\title{
A Literature Review of Infection with ESKAPE Pathogens in Oral and Maxillofacial Region
}

\author{
Sang-Yeap Park, Jae-Seek You, Seong-Yong Moon, Ji-Su Oh, Hae-In Choi, Gyeo-Woon Jung \\ Department of Oral and Maxillofacial Surgery, School of Dentistry, Chosun University, Gwangju, Korea
}

Received July 19, 2021

Revised August 19, 2021

Accepted August 20, 2021

\section{Correspondence to:}

Jae-Seek You

Department of Oral and Maxillofacial

Surgery, School of Dentistry, Chosun

University, 303 Pilmun-daero, Dong-gu,

Gwangju 61452, Korea

Tel: +82-62-220-3816

Fax: +82-62-222-3810

E-mail:applit375@chosun.ac.kr

https://orcid.org/0000-0001-7638-9583

This study was supported by research fund from Chosun University (2019).
Odontogenic infection in the oral and maxillofacial regions caused by bacteria (mostly of oral origin) is one of the most common diseases encountered by dentists. Localized infection can easily be treated with incision and drainage followed by antibiotics. Emergence of multidrug resistant (MDR) bacteria called "Superbacteria" has become one of the serious problems in modern society, due to its small window of opportunity for treatment and high casualty. The acronym "ESKAPE", encompassing the common and serious MDR pathogens stand for Enterococcus faecium, Staphylococcus aureus, Klebsiella pneumoniae, Acinetobacter baumannii, Pseudomonas aeruginosa and Enterobacter spp. Literature search was performed in Medline, PubMed and Google Scholar ranging from 2012 to 2020. ESKAPE patient's infection period was longer than that of non-ESKAPE group, and the treatment method due to antibiotic resistance was also complicated. The purpose of this study is to investigate infection caused by ESKAPE pathogens in the oral and maxillofacial regions through literature review and to inform dental surgeons of the danger of ESKAPE pathogens and to suggest viable treatment options. Many studies worldwide reported infections associated with ESKAPE pathogens, but only limited number of studies targeted infection in oral and maxillofacial regions. Further research is required with more data on ESKAPE bacteria and their infection, especially in oral and maxillofacial regions.

Key Words: Abscess; ESKAPE; Infections; Methicillin-resistant Staphylococcus aureus; Osteomyelitis

\section{INTRODUCTION}

Odontogenic infection in the oral and maxillofacial regions caused by bacteria (mostly of oral origin) is one of the most common diseases encountered by dentists [1]. Localized infection can easily be treated with incision and drainage (I\&D) followed by antibiotics. However, it can spread rapidly to other spaces and cause severe problems such as sepsis, airway obstruction, necrotizing fasciitis and mediastinitis [2-4]. Odontogenic infection is polymicrobial in nature, comprising of various facultative anaerobes, such as the viridans group, streptococcus species, and strict anaerobes, especially anaerobic cocci, prevotella and fusobacterium species [5,6]. Emergence of multidrug resistant (MDR) bacteria called "Superbacteria" has become one of the serious problems in modern society, due to its small window of opportunity for treatment and high mortality. The acronym "ESKAPE", encompassing the common MDR pathogens stands for Enterococcus faecium, Staphylococcus aureus, Klebsiella pneumoniae, Acinetobacter baumannii, Pseudomonas aeruginosa and Enterobacter spp. [7] and they are responsible for causing nosocomial and opportunistic infections in hospitals. ESKAPE species has characteristic of healthcare-acquired infection that can pose a great threat to human health and become increasingly more resistant to commonly used antibiotics [8]. These resistant 
bacteria are mainly responsible for pneumonia, bloodstream infection, sepsis, surgical site infection (SSI), and urinary tract infection [9]. Likewise, there are many medical reviews of ESKAPE pathogens, but few in oral and maxillofacial regions. The purpose of this study is to investigate infection caused by ESKAPE pathogens in the oral and maxillofacial regions through literature review and to inform dental surgeons of the danger of ESKAPE pathogens and to suggest viable treatment options.

\section{MATERIALS AND METHODS}

Literature search was performed in Medline, PubMed and Google Scholar ranging from 2012 to 2020, using keywords, including "ESKAPE pathogens", "K. pneumoniae", "A. baumannii" "P. aeruginosa", "S. aureus", "E. faecium", "Enterobacter species", and "Infection in orofacial or oral and maxillofacial". The criteria included types of study, clinical diagnosis, bacteria species (especially, ESKAPE pathogens), treatment approach, respect of infections, characteristics of patients and other clinical parameters. In addition, references of all retrieved articles were checked for further relevant literature with this topic.

\section{RESULT}

This study found 16 articles from 2012 to 2020 in Medline, PubMed and Google Scholar and performed comparative analysis. As shown in Tables 1 and 2, articles discussed various bacterial infection on different sites. This study specifically focused on ESKAPE bacteria. Of the 16 articles reviewed in this paper, 11 articles studied medical and systemic infection, and only 5 articles studied oral and maxillofacial infection, mostly of dental origin (Tables 1, 2). Empirical antibiotics were first administered, and culture was performed to identify bacteria type and antibiotic sensitivity. With the result of culture test, carbapenem was most commonly used, and vancomycin was mainly used in $E$. faecium and $S$. aureus infection. In addition, it was confirmed that postoperative infection occurred in 31\% of the papers and 68\% showed that the infected patients stayed in the hospital for a long time, which continued to increase the risk of infection. Analysis showed that ESKAPE infection route was mainly nosocomial infection rather than community infection. The healing period of patient with ESKAPE was longer than that of non-ESKAPE group, and the treatment method was also complicated because of its antibiotic resistance.

\section{DISCUSSION}

The problem of infection has continued to exist even after the introduction of antibiotics and the emergence of antibiotic resistant bacteria (ARB) has become a worldwide issue that threatens individual healthcare, economic and social welfare [10-12]. Despite increased efforts in recent years, the problem of ARB continues to grow [9,13]. Especially, ESKAPE pathogens are mostly resistant to antibiotics and the risk of infection is growing due to mechanisms including inactivation or alteration of antimicrobial molecule, modification of the site of action, inhibition of cell membrane function, and reduction of antibiotic penetration/ accumulation $[11,14,15]$. Extensive resistance to antibiotic requires discreet infection prevention, development of new antibiotics and treatment methods. Most previous studies reported on systemic infections in medical field caused by ESKAPE pathogens and different antibiotics that effectively treat ARB.

Only a few researches have been performed on ESKAPE infection and treatment of oral and maxillofacial region. Among the various bacteria, $S$. aureus was mainly observed among ESKAPE pathogens. S. aureus consists normal human flora, including nose, oral cavity and perineum and may lead to opportunistic infection in both hospital and community [16]. With regards to oral infections, a review on orofacial bacterial infections diseases reported that, $S$. aureus was also associated with a bullous type of facial infections around the nose and the mouth of patients with dry mouth and denture $[17,18]$. In the nares, $S$. aureus interacts with nasal mucosa's epithelial cell ligands and colonizes the area [19]. Hands serve as the main transporter from the nose to other locations in the body [20]. Oral cavity has been identified as a significant reservoir of $S$. aureus in an acute hospital by healthcare worker, extensive patient [21]. $S$. aureus is an opportunistic pathogen that is not a problem in normal situation. Traditionally, infections have 


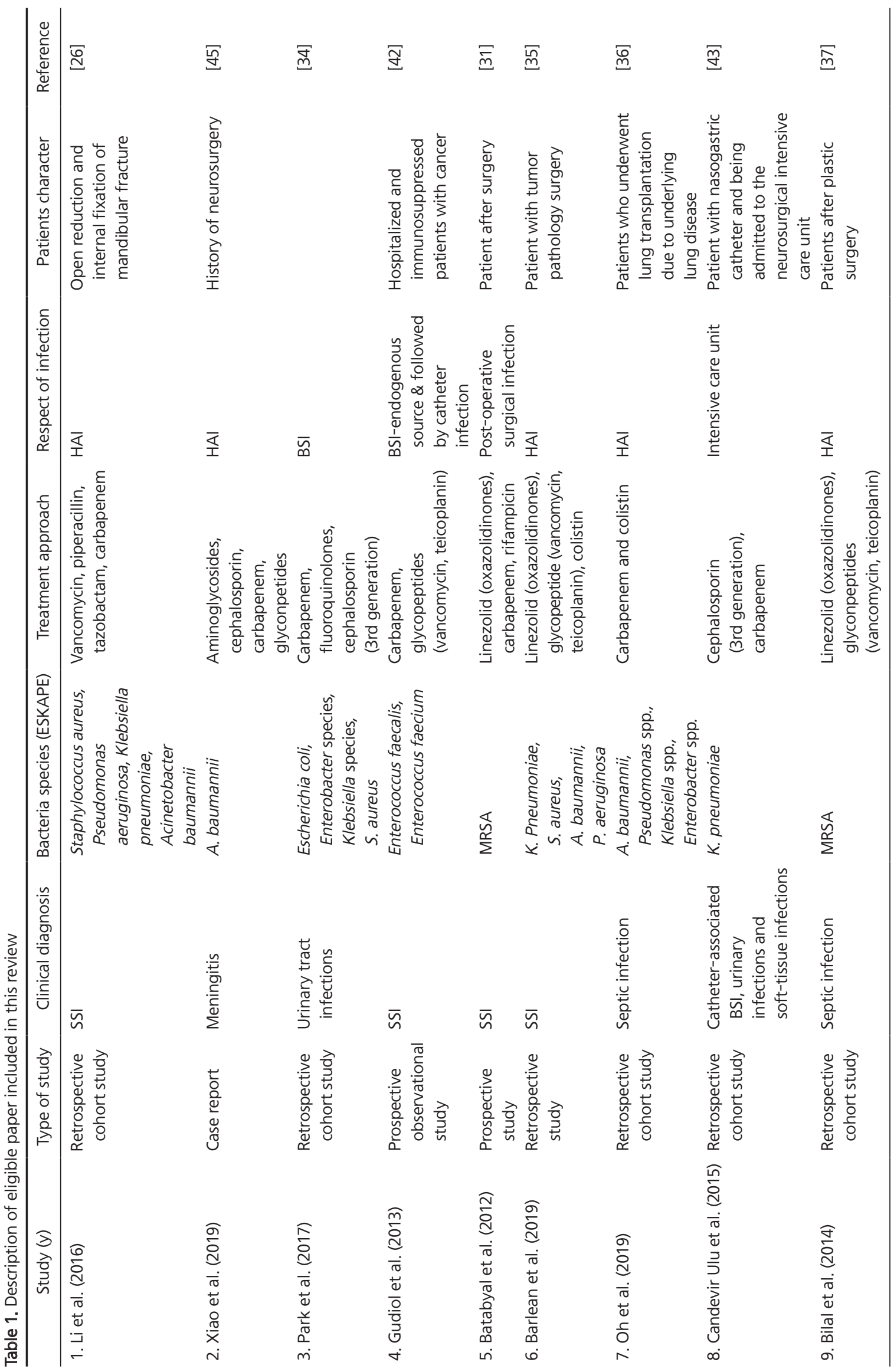




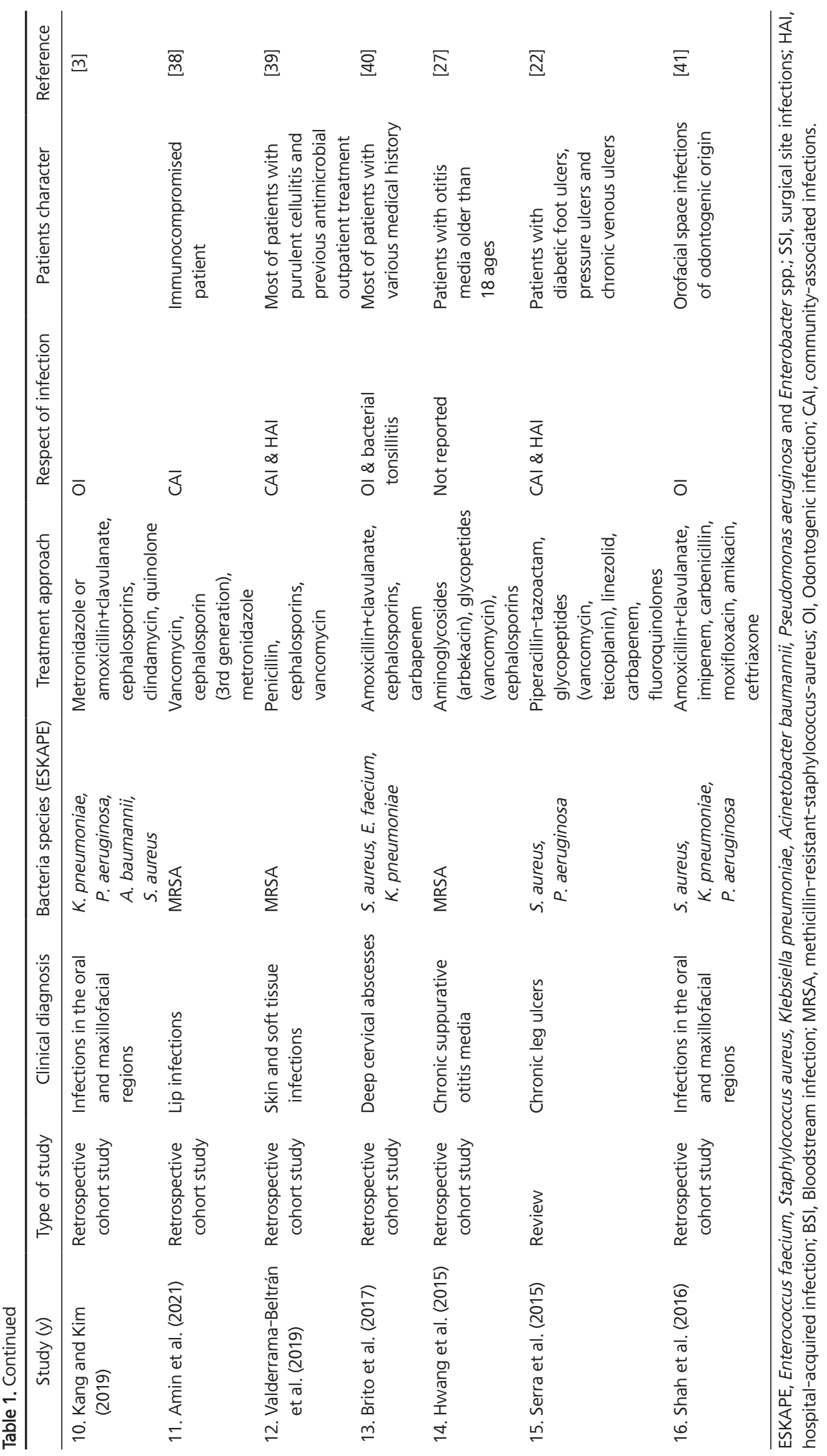




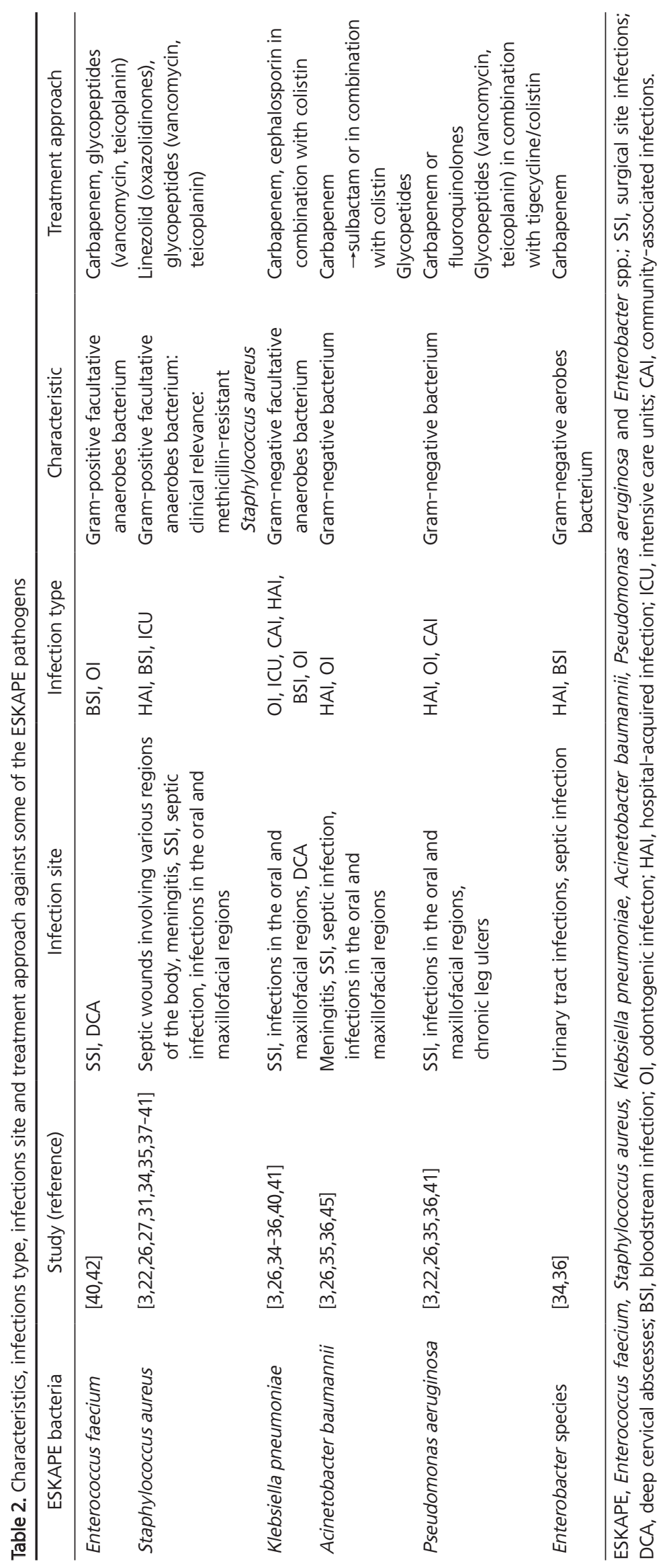


responded well to penicillin treatment [11,22].

However, methicillin resistance should also be taken into consideration in clinical situation. Most common methicillin-resistant $S$. aureus (MRSA) infections in oral and maxillofacial area were postoperative to primary resections with reconstructions using free flap [23]. A few cases of osteomyelitis of jaws [24,25] and SSI by mandible fracture [26] were also reported to be related to $S$. aureus infection.

These MRSA strains are resistant to many antibiotics such as $\beta$-lactam antibiotic, aminoglycosides, macrolides, and choloramphenicol [27]. In most cases, glycopeptide antibiotics, such as vancomycin and teicoplanin, are used as first-line of antibiotics for of MRSA infections [11,28,29]. Emergence of vancomycin-resistant $S$. aureus has also been reported $[22,30]$. In order to treat MRSA with low sensitivity to vancomycin, costly medication such as linezolid, daptomycin, etc. can be prescribed [31].

$K$. pneumoniae is a gram-negative facultative anaerobes bacteria and is known to asymptomatically colonize the skin, mouth, respiratory and gastric intestinal tract [32,33]. Multiple studies reported that $S$. aureus and $K$. pneumoniae may cause bacteremia, septicemia, soft tissue infections, endocarditis, bone infections, pneumonia, blood stream infection (BSI), and SSI [3,22,26,27,31,34-43]. The ability of $K$. pneumoniae to produce extended spectrum beta-lactamase makes it resistant to all beta-lactam antibiotics except carbapenem, thereby making carbapenem preferable treatment option [44].

Like other bacteria, infection of $A$. baumannii is problematic, because of limited therapeutic options due to increasing resistance to antibiotics groups and the capacity to persist in the hospital environment, resulting in outbreaks $[45,46]$. The most common clinical manifestations of $A$. baumannii are ventilator-associated pneumonia and bloodstream infection. A. baumannii can also colonize the skin, wounds, respiratory tract and gastrointestinal tract $[47,48]$.

Carbapenems are conventionally used to treat persistent infections caused by gram-negative bacteria and were first administered to other ESKAPE bacteria except for MRSA, and it can be used in conjunction with colistin to treat $A$. baumannii and $P$. aeruginosa $[11,49]$. In patient with $P$. aeruginosa infection, infection of imipenem, ceftazidime, ciprofloxacin or piperacillin resistant bacteria is associated with significantly prolonged hospitalization and increased chance of secondary bacteremia [50].

Various antibiotics are being administered depending on the bacteria, and antibiotic resistance is developed accordingly. Inadequate use of antibiotics increases the risk of drug resistance, leading to impairment of a patient's condition. For effective treatment of ARB, early diagnosis is essential [51].

Early detection of causative agents and selection of corresponding antibiotic is stressed [12,52]. Empirical prescription of antibiotics and antibiotic-susceptibility test (culture test) should be performed [53]. Based on the results of culture testing, non-resistant and sensitive antibiotics must be selected and treated. Evaluating the patient's clinical condition is also important. If other bacteria are found in culture test, it is necessary to see if they respond to the currently prescribed antibiotics. In other words, the most appropriate antibiotics should be selected in cooperation with the Infectious Disease Department. Many papers suggest that immunosuppressed patients are more susceptible to infection [32,33]. An article studied patients with human immunodeficiency virus [38] and another article studied with diabetes mellitus $[22,40]$. In diabetic patients, certain infections were more predominant, and some outbreak appeared almost exclusively. Diabetes was also associated with increasing severity of the infections and increased chance of complication. Diabetic patients had compromised immune system in several aspects [54]. Accordingly, systemic conditions can influence the progress of infection, and patient's comprehensive health condition is crucial in the course of treatment.

However, according to other studies, the frequency of infection by ESKAPE pathogens is not significantly different from that of the non-ESKAPE group in terms of gender, the presence of systemic disease, and the frequency of occurrence by infection site $[55,56]$. However, it was found that the treatment period was longer in patients with systemic diseases of ESKAPE-group than in patient of non-ESKAPE group.

This study confirmed high rate of SSI and BSI by ESKAPE pathogens. Fifty percentage of the studies reported healthcare-associated infection that can be also affected adversely to patients who admitted to the intensive care units. 
Most nosocomial infections can be derived from exogenous sources and transferred by either direct or indirect contact between patient, healthcare workers and contaminated objects [13]. As a result, clinicians should be mindful to prevent infection. Especially, dentists should take proper precautions to prevent cross contamination of bacteria in the dental clinic and hospitals, where infection is easily spread by aerosols. Infection by ESKAPE pathogens is associated with a longer treatment period, higher cost of care and a higher mortality rate compared to that of non-ESKAPE pathogens $[57,58]$. A systematic approach to surveillance, infection prevention, antimicrobial stewardship and clinical guidelines ensures best practice for infection control and reduces the spread of antimicrobial resistance [59]. Directing attention to the ESKAPE pathogens can better address the broader challenges of MDR.

We expect some difficulties in our study and acknowledge some limitations. First, literature reviewed in this paper did not have consensus over the definition of the outcomes. Following a single published guidance on assessment of outcomes of different infection sites should provide more statistically meaningful conclusions. Second, there was a limitation to the fundamental analysis due to lack of the number of papers on infection focused with ESKAPE pathogens in the oral and maxillofacial regions, and the gold standard treatment option cannot be proffered. Third, due to the variety of infection areas and variety of antibiotics empirically prescribed for treatment, the effect of antibiotic resistance could not be properly analyzed. Fourth, not all identified pathogens were tested against the administered antibiotics and it is difficult to analyze and compare various antibiotic resistance rates by bacteria. In the future, comparing different strains applied with the same antibiotics will yield a more meaningful conclusion. In addition, there were limitations in classifying risk factors.

\section{CONCLUSION}

Many studies worldwide reported infections associated with ESKAPE pathogens, but only limited number of studies targeted infection in oral and maxillofacial regions. Infection by ESKAPE bacteria can have fatal consequences if the cause of the infection is not properly identified and characterized in an early stage. Further research is required with more data on ESKAPE bacteria and their infection, especially in oral and maxillofacial regions.

\section{CONFLICT OF INTEREST}

No potential conflict of interest relevant to this article was reported.

\section{ORCID}

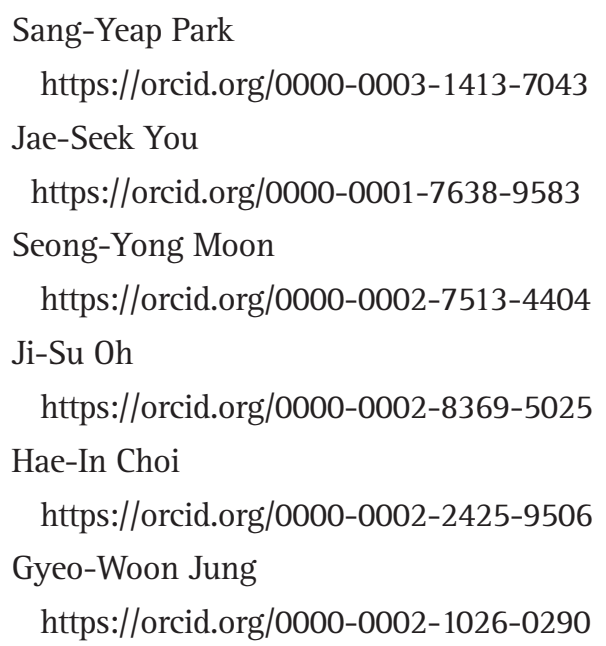

\section{REFERENCES}

1. Dahlén G. Microbiology and treatment of dental abscesses and periodontal-endodontic lesions. Periodontol 2000 2002;28:206239.

2. Bertossi D, Barone A, Iurlaro A, et al. Odontogenic orofacial infections. J Craniofac Surg 2017;28:197-202.

3. Kang SH, Kim MK. Antibiotic sensitivity and resistance of bacteria from odontogenic maxillofacial abscesses. J Korean Assoc Oral Maxillofac Surg 2019;45:324-331.

4. Seppänen L, Lauhio A, Lindqvist C, Suuronen R, Rautemaa R. Analysis of systemic and local odontogenic infection complications requiring hospital care. J Infect 2008;57:116-122.

5. Shweta, Prakash SK. Dental abscess: a microbiological review. Dent Res J (Isfahan) 2013;10:585-591.

6. Nair PN. Pathogenesis of apical periodontitis and the causes of endodontic failures. Crit Rev Oral Biol Med 2004;15:348-381.

7. Rice LB. Federal funding for the study of antimicrobial resistance in nosocomial pathogens: no ESKAPE. J Infect Dis 2008;197:1079-1081.

8. Stavropoulou E, Tsigalou C, Bezirtzoglou E. Spreading of antimicrobial resistance across clinical borders. Erciyes Med J 2019;41:238-243.

9. Michiels JE, Van den Bergh B, Verstraeten N, Fauvart M, Michiels 
J. In vitro emergence of high persistence upon periodic aminoglycoside challenge in the ESKAPE pathogens. Antimicrob Agents Chemother 2016;60:4630-4637.

10. Roca I, Akova M, Baquero F, et al. The global threat of antimicrobial resistance: science for intervention. New Microbes New Infect 2015;6:22-29.

11. Santajit S, Indrawattana N. Mechanisms of antimicrobial resistance in ESKAPE pathogens. Biomed Res Int 2016;2016:2475067.

12. Founou RC, Founou LL, Essack SY. Clinical and economic impact of antibiotic resistance in developing countries: a systematic review and meta-analysis. PLoS One 2017;12:e0189621.

13. World Health Organization. Antimicrobial resistance: global report on surveillance. Geneva: World Health Organization; 2014.

14. Ma YX, Wang CY, Li YY, et al. Considerations and caveats in combating ESKAPE pathogens against nosocomial infections. Adv Sci (Weinh) 2019;7:1901872.

15. Pendleton JN, Gorman SP, Gilmore BF. Clinical relevance of the ESKAPE pathogens. Expert Rev Anti Infect Ther 2013;11:297308.

16. NeVille-Swensen M, Clayton M. Outpatient management of community-associated methicillin-resistant Staphylococcus aureus skin and soft tissue infection. J Pediatr Health Care 2011;25:308315.

17. Irani S. Orofacial bacterial infectious diseases: an update. J Int Soc Prev Community Dent 2017;7(Suppl 2):S61-S67.

18. Murakami M, Nishi Y, Seto K, Kamashita Y, Nagaoka E. Dry mouth and denture plaque microflora in complete denture and palatal obturator prosthesis wearers. Gerodontology 2015;32:188194.

19. Sakr A, Brégeon F, Mège JL, Rolain JM, Blin O. Staphylococcus aureus nasal colonization: an update on mechanisms, epidemiology, risk factors, and subsequent infections. Front Microbiol 2018;9:2419.

20. Reagan DR, Doebbeling BN, Pfaller MA, et al. Elimination of coincident Staphylococcus aureus nasal and hand carriage with intranasal application of mupirocin calcium ointment. Ann Intern Med 1991;114:101-106.

21. Kearney A, Kinnevey P, Shore A, et al. The oral cavity revealed as a significant reservoir of Staphylococcus aureus in an acute hospital by extensive patient, healthcare worker and environmental sampling. J Hosp Infect 2020;105:389-396.

22. Serra R, Grande R, Butrico L, et al. Chronic wound infections: the role of Pseudomonas aeruginosa and Staphylococcus aureus. Expert Rev Anti Infect Ther 2015;13:605-613.

23. Avery CM, Ameerally P, Castling B, Swann RA. Infection of surgical wounds in the maxillofacial region and free flap donor sites with methicillin-resistant Staphylococcus aureus. Br J Oral Maxillofac Surg 2006;44:217-221.

24. Burduk PK, Skorek A, Stankiewicz C, Dalke K. Chronic, recurrent, progressive osteomyelitis of the maxilla caused by methicillinresistant Staphylococcus epidermidis: a therapeutic dilemma. J Oral Maxillofac Surg 2010;68:2012-2015.

25. Tuzuner-Oncul AM, Ungor C, Dede U, Kisnisci RS. Methicillinresistant Staphylococcus aureus (MRSA) osteomyelitis of the mandible. Oral Surg Oral Med Oral Pathol Oral Radiol Endod
2009; 107:e1-e4.

26. Li Z, Zhou Z, Li P, Zeng W, Qing H, Tang W. Retrospective study on multidrug-resistant bacterium infections after rigid internal fixation of mandibular fracture. J Oral Maxillofac Surg 2016;74:770-777.

27. Hwang JH, Lee JH, Hwang JH, et al. Comparison of arbekacin and vancomycin in treatment of chronic suppurative otitis media by methicillin resistant Staphylococcus aureus. J Korean Med Sci 2015;30:688-693.

28. Lourtet-Hascoët J, Félicé MP, Bicart-See A, Bouige A, Giordano G, Bonnet E. Species and antimicrobial susceptibility testing of coagulase-negative staphylococci in periprosthetic joint infections. Epidemiol Infect 2018;146:1771-1776.

29. Lee HJ, You JS. Postoperative infection caused by methicillinresistant coagulase-negative Staphylococci in the oral and maxillofacial region: a case report. J Oral Med Pain 2020;45:17-21.

30. Gurusamy KS, Koti R, Toon CD, Wilson P, Davidson BR. Antibiotic therapy for the treatment of methicillin-resistant Staphylococcus aureus (MRSA) in non surgical wounds. Cochrane Database Syst Rev 2013;(11):CD010427.

31. Batabyal B, Biswas S, Chakraborty S, Desai PD, De Sarkar N. Prevalence and drug sensitivity pattern of Staphylococcus aureus in post-operative surgical oral \& maxillofacial infections. Int J Life Sci Pharma Res 2012;2:150-158.

32. Garbino J, Inoubli S, Mossdorf E, et al. Respiratory viruses in HIV-infected patients with suspected respiratory opportunistic infection. AIDS 2008;22:701-705.

33. Shu Kurizky P, Dos Santos Neto LL, Barbosa Aires R, Henrique da Mota LM, Martins Gomes C. Opportunistic tropical infections in immunosuppressed patients. Best Pract Res Clin Rheumatol 2020;34:101509.

34. Park JJ, Seo YB, Lee J. Antimicrobial susceptibilities of Enterobacteriaceae in community-acquired urinary tract infections during a 5-year period: a single hospital study in Korea. Infect Chemother 2017;49:184-193.

35. Barlean MC, Balcos C, Bobu LI, et al. Microbiological evaluation of surgical site infections in the Clinic of Oral and Maxillofacial Surgery of the Sf.Spiridon Clinical Hospital in Iasi, Romania. Rev Chim 2019;70:4077-4082.

36. Oh DH, Kim YC, Kim EJ, et al. Multidrug-resistant Acinetobacter baumannii infection in lung transplant recipients: risk factors and prognosis. Infect Dis (Lond) 2019;51:493-501.

37. Bilal M, Janan A, Irfanullah MA. Occurrence of methicillin resistant staphylococcus aureus (MRSA) in patients requiring reconstructive surgery. Pak J Surg 2014;30:301-305.

38. Amin D, Satishchandran S, Drew S, Abramowicz S. Diagnosis and treatment of lip infections. J Oral Maxillofac Surg 2021;79:133140.

39. Valderrama-Beltrán S, Gualtero S, Álvarez-Moreno C, et al. Risk factors associated with methicillin-resistant Staphylococcus aureus skin and soft tissue infections in hospitalized patients in Colombia. Int J Infect Dis 2019;87:60-66.

40. Brito TP, Hazboun IM, Fernandes FL, et al. Deep neck abscesses: study of 101 cases. Braz J Otorhinolaryngol 2017;83:341-348.

41. Shah A, Ramola V, Nautiyal V. Aerobic microbiology and culture 
sensitivity of head and neck space infection of odontogenic origin. Natl J Maxillofac Surg 2016;7:56-61.

42. Gudiol C, Ayats J, Camoez M, et al. Increase in bloodstream infection due to vancomycin-susceptible Enterococcus faecium in cancer patients: risk factors, molecular epidemiology and outcomes. PLoS One 2013;8:e74734.

43. Candevir Ulu A, Kurtaran B, Inal AS, et al. Risk factors of carbapenem-resistant Klebsiella pneumoniae infection: a serious threat in ICUs. Med Sci Monit 2015;21:219-224.

44. Maslikowska JA, Walker SA, Elligsen M, et al. Impact of infection with extended-spectrum $\beta$-lactamase-producing Escherichia coli or Klebsiella species on outcome and hospitalization costs. J Hosp Infect 2016;92:33-41.

45. Xiao J, Zhang C, Ye S. Acinetobacter baumannii meningitis in children: a case series and literature review. Infection 2019; 47:643-649.

46. Abbo A, Navon-Venezia S, Hammer-Muntz 0, Krichali T, Siegman-Igra Y, Carmeli Y. Multidrug-resistant Acinetobacter baumannii. Emerg Infect Dis 2005;11:22-29.

47. Maragakis LL, Perl TM. Acinetobacter baumannii: epidemiology, antimicrobial resistance, and treatment options. Clin Infect Dis 2008;46:1254-1263.

48. Park SY, Choo JW, Kwon SH, et al. Risk factors for mortality in patients with Acinetobacter baumannii bacteremia. Infect Chemother 2013;45:325-330.

49. Boucher HW, Talbot GH, Bradley JS, et al. Bad bugs, no drugs: no ESKAPE! An update from the Infectious Diseases Society of America. Clin Infect Dis 2009;48:1-12.

50. Paramythiotou E, Lucet JC, Timsit JF, et al. Acquisition of multidrug-resistant Pseudomonas aeruginosa in patients in intensive care units: role of antibiotics with antipseudomonal activity. Clin
Infect Dis 2004;38:670-677.

51. Moradigaravand D, Palm M, Farewell A, Mustonen V, Warringer J, Parts L. Prediction of antibiotic resistance in Escherichia coli from large-scale pan-genome data. PLoS Comput Biol 2018; 14:e1006258.

52. Zhen X, Lundborg CS, Sun X, Hu X, Dong H. Economic burden of antibiotic resistance in ESKAPE organisms: a systematic review. Antimicrob Resist Infect Control 2019;8:137.

53. Giulieri SG, Tong SYC, Williamson DA. Using genomics to understand meticillin- and vancomycin-resistant Staphylococcus aureus infections. Microb Genom 2020;6:e000324.

54. Joshi N, Caputo GM, Weitekamp MR, Karchmer AW. Infections in patients with diabetes mellitus. N Engl J Med 1999;341:19061912.

55. Takai S, Kuriyama T, Yanagisawa M, Nakagawa K, Karasawa T. Incidence and bacteriology of bacteremia associated with various oral and maxillofacial surgical procedures. Oral Surg Oral Med Oral Pathol Oral Radiol Endod 2005;99:292-298.

56. Jang SJ, Lee YG, Ahn Y, Leem DH, Baek JA, Shin HK. A clinicostastical study of oral and maxillofacial infected patients for the last 5 years. J Korean Assoc Oral Maxillofac Surg 2006;32:401409.

57. Veronez B, de Matos FP, Monnazzi MS, Sverzut AT, Sverzut CE, Trivellato AE. Maxillofacial infection. A retrospective evaluation of eight years. Braz J Oral Sci 2014;13:98-103.

58. Marturano JE, Lowery TJ. ESKAPE pathogens in bloodstream infections are associated with higher cost and mortality but can be predicted using diagnoses upon admission. Open Forum Infect Dis 2019;6:ofz503.

59. Fishman N. Antimicrobial stewardship. Am J Med 2006;119(6 Suppl 1):S53-S61; discussion S62-S70. 